\title{
Dynamics and oscillations of models for differential equations with delays
}

\author{
Mohsen Miraoui ${ }^{1,2}$ and Dušan D. Repovš ${ }^{3,4,5 *}$
}

\author{
"Correspondence: \\ dusan.repovs@guest.arnes.si \\ ${ }^{3}$ Faculty of Education, University of \\ Ljubljana, Ljubljana, Slovenia \\ ${ }^{4}$ Faculty of Mathematics and \\ Physics, University of Ljubljana, \\ Ljubljana, Slovenia \\ Full list of author information is \\ available at the end of the article
}

\begin{abstract}
By developing new efficient techniques and using an appropriate fixed point theorem, we derive several new sufficient conditions for the pseudo almost periodic solutions with double measure for some system of differential equations with delays. As an application, we consider certain models for neural networks with delays.

MSC: 34K14; 35B15; 82C32

Keywords: Dynamics; Oscillation; Periodic solution; Time delay; Stability; Ergodicity; Double measure; Fixed point theorem; Neural network
\end{abstract}

\section{Introduction}

Existence of periodic, almost periodic, and pseudo almost periodic solutions of differential equations has great significance and is therefore an important problem. Such dynamics can be found in electronic circuits and many other physical and biological systems (see $[3,6,9,18-21,23,26])$. Ezzinbi et al. [5] introduced a new and powerful measure-theoretic method to resolve this open problem. Since then, this method has been used for various classes of evolution equations as well as stochastic differential equations and has become very popular.

The notion of measure pseudo almost periodicity was first introduced by Blot et al. [5] (see also $[1,8,12,13,15-17,27])$. Obviously, these new results generalize the earlier work of Diagana [10]. Recently, Diagana et al. [11] have introduced the notion of double measure pseudo almost periodicity as a generalization of the measure pseudo almost periodicity. We note that this generalized concept coincides with the latter one (take $\mu \equiv v$ ).

In this paper, by applying an appropriate fixed point theorem, we derive some conditions which ensure the existence, the exponential stability, and the uniqueness of $(\mu, v)$-pap solutions of the following models with delays:

$$
\begin{aligned}
x_{i}^{\prime}(t)= & -c_{i}(t) x_{i}(t)+\sum_{j=1}^{n} d_{i j}(t) f_{j}\left(t, x_{j}(t)\right)+\sum_{j=1}^{n} a_{i j}(t) g_{j}\left(t, x_{j}\left(t-\tau_{i j}\right)\right) \\
& +\sum_{j=1}^{n} \sum_{l=1}^{n} b_{i j l}(t) h_{j}\left(t, x_{j}\left(t-\sigma_{i j}\right)\right) h_{l}\left(t, x_{l}\left(t-v_{i j}\right)\right)+I_{i}(t),
\end{aligned}
$$

(c) The Author(s) 2020. This article is licensed under a Creative Commons Attribution 4.0 International License, which permits use, sharing, adaptation, distribution and reproduction in any medium or format, as long as you give appropriate credit to the original author(s) and the source, provide a link to the Creative Commons licence, and indicate if changes were made. The images or other third party material in this article are included in the article's Creative Commons licence, unless indicated otherwise in a credit line to the material. If material is not included in the article's Creative Commons licence and your intended use is not permitted by statutory regulation or exceeds the permitted use, you will need to obtain permission directly from the copyright holder. To view a copy of this licence, visit http://creativecommons.org/licenses/by/4.0/. 


$$
x_{i}(s)=\varphi_{i}(s), \quad s \in(-\theta, 0], \quad i \in\{1, \ldots, n\},
$$

where functions

$$
c_{i}, I_{i}, d_{i j}, a_{i j}, b_{i j l}: \mathbb{R} \rightarrow \mathbb{R} \quad \text { and } \quad f_{j}, g_{j}, h_{j}: \mathbb{R} \times \mathbb{R} \rightarrow \mathbb{R}, \quad i, j, l \in\{1, \ldots, n\}
$$

are continuous and $\tau_{i j}$, $\sigma_{i j}$, and $v_{i j}$ are positive constants.

The paper is organized as follows: in Sect. 2 we collect key definitions, examples, and basic results. In Sect. 3 we discuss the existence, the stability, and the uniqueness of double measure pseudo almost periodic solutions of system (1.1). Finally, in Sect. 4 we present an application which illustrates the effectiveness of our results.

\section{Preliminaries}

Definition 2.1 (see [5]) Let $f$ be a continuous function on $\mathbb{R}$ with values in $\mathbb{R}^{n}$. Then $f$ is said to be almost periodic, denoted by $f \in \mathcal{A P}\left(\mathbb{R}, \mathbb{R}^{n}\right)$, if for all $\varepsilon>0$, there exists a number $l(\varepsilon)>0$ such that every interval $I$ of length $l(\varepsilon)$ contains a point $\tau \in \mathbb{R}$ with the property that

$$
\|f(t+\tau)-f(t)\|<\varepsilon \quad \text { for all } t \in \mathbb{R} .
$$

The space $\mathcal{A P}\left(\mathbb{R}, \mathbb{R}^{n}\right)$ equipped with the norm

$$
\|f\|_{\infty}:=\max _{1 \leq i \leq n} \sup _{t \in \mathbb{R}}\left|f_{i}(t)\right|
$$

is then a Banach space. Let $\mathcal{B}$ be the Lebesque $\sigma$-field on $\mathbb{R}$ and define a collection $\mathcal{M}$ of measures on $\mathcal{B}$

$$
\begin{aligned}
\mathcal{M}= & \{\mu \text { is a positive measure on } \mathcal{B} ; \\
& \mu(\mathbb{R})=+\infty, \text { and } \mu([s, t])<\infty, \text { for all } s, t \in \mathbb{R}, s \leq t\} .
\end{aligned}
$$

Let $X$ be a Banach space, and denote by $\mathcal{B C}(\mathbb{R}, X)$ the Banach space of bounded continuous functions from $\mathbb{R}$ to $X$, equipped with the supremum norm $\|f\|_{\infty}=\sup _{t \in \mathbb{R}}\|f(t)\|$. In order to be able to introduce double measure pseudo almost periodic functions, we need the following ergodic spaces:

$$
\mathcal{E}\left(\mathbb{R}, \mathbb{R}^{n}, \mu, v\right):=\left\{f \in \mathcal{B C}\left(\mathbb{R}, \mathbb{R}^{n}\right): \lim _{z \rightarrow \infty} \frac{1}{v([-z, z])} \int_{-z}^{z}\|f(t)\| d \mu(t)=0\right\}
$$

and

$$
\mathcal{E}\left(\mathbb{R}, \mathbb{R}^{n}, \mu\right):=\mathcal{E}\left(\mathbb{R}, \mathbb{R}^{n}, \mu, \mu\right)=\left\{f \in \mathcal{B C}\left(\mathbb{R}, \mathbb{R}^{n}\right): \lim _{z \rightarrow \infty} \frac{1}{\mu([-z, z])} \int_{-z}^{z}\|f(t)\| d \mu(t)=0\right\} .
$$

Definition 2.2 (see [11]) If $\mu, v \in \mathcal{M}$, then $f \in \mathcal{B C}\left(\mathbb{R}, \mathbb{R}^{n}\right)$ is said to be $(\mu, v)$-pseudo almost periodic, abbreviated as $(\mu, v)$-pap, denoted by $f \in \mathcal{P} \mathcal{A} \mathcal{P}\left(\mathbb{R}, \mathbb{R}^{n}, \mu, v\right)$, if there exists a decomposition

$$
f=g+\varphi, \quad \text { where } \varphi \in \mathcal{E}\left(\mathbb{R}, \mathbb{R}^{n}, \mu, v\right) \text { and } g \in \mathcal{A P}\left(\mathbb{R}, \mathbb{R}^{n}\right) \text {. }
$$


We also introduce the following notation $\mathcal{P} \mathcal{A} \mathcal{P}\left(\mathbb{R}, \mathbb{R}^{n}, \mu\right):=\mathcal{P} \mathcal{A} \mathcal{P}\left(\mathbb{R}, \mathbb{R}^{n}, \mu, \mu\right)$.

Definition 2.3 (see [11]) If $\mu, v \in \mathcal{M}$ and $f(t, u): \mathbb{R} \times \mathbb{R} \rightarrow \mathbb{R}^{n}$ is continuous, then $f(t, u)$ is said to be $(\mu, v)$-pseudo almost periodic in $t$, uniformly with respect to $u$, abbreviated as $(\mu, v)$-papu, denoted by $f \in \mathcal{P} \mathcal{A P U}\left(\mathbb{R} \times \mathbb{R}, \mathbb{R}^{n}, \mu, v\right)$, if

$$
f=g+h, \quad \text { where } g \in \mathcal{A P U}\left(\mathbb{R} \times \mathbb{R}, \mathbb{R}^{n}\right) \text { and } h \in \mathcal{E} U\left(\mathbb{R} \times \mathbb{R}, \mathbb{R}^{n}, \mu\right) .
$$

Example 2.1 Let $\mu \in \mathcal{M}$ and

$$
G(t)=[\sin (t)+\sin (\sqrt{2} t)] \cos (x)+\frac{\sin (x)}{1+t^{2}}, \quad t \in \mathbb{R} .
$$

Then $G \in \mathcal{P} \mathcal{A} \mathcal{P U}(\mathbb{R} \times \mathbb{R}, \mathbb{R}, \mu)$.

We shall need the following two conditions:

(M.1) For every measure $\mu \in \mathcal{M}$ and every $\tau \in \mathbb{R}$, there exist $\beta>0$ and a bounded interval $I$ such that, for every $A \in \mathcal{B}$,

$$
A \cap I=\emptyset \quad \Longrightarrow \quad \mu_{\tau}(A):=\mu(\{a+\tau: a \in A\}) \leq \beta \mu(A) .
$$

(M.2) Measures $\mu, v \in \mathcal{M}$ satisfy the following condition:

$$
\limsup _{r \rightarrow \infty} \frac{\mu([-r, r])}{v([-r, r])}<\infty
$$

Lemma 2.2 (see [11]) Let $\mu, v \in \mathcal{M}$ and suppose that conditions (M.1) and (M.2) hold. Then

- decomposition (2.1) above is unique;

- $\left(\mathcal{P} \mathcal{A P}\left(\mathbb{R}, \mathbb{R}^{n}, \mu, v\right),\|\cdot\|_{\infty}\right)$ is a Banach space; and

- $\mathcal{P} \mathcal{A} \mathcal{P}\left(\mathbb{R}, \mathbb{R}^{n}, \mu, v\right)$ is translation invariant.

\section{Double measure pseudo almost periodic solutions}

We introduce the following notations:

$$
\begin{array}{ll}
\sup _{t \in \mathbb{R}}\left\{\left|d_{i j}(t)\right|\right\}:=\bar{d}_{i j}, & \sup _{t \in \mathbb{R}}\left\{\left|I_{i}(t)\right|\right\}:=\bar{I}_{i}, \\
\sup _{t \in \mathbb{R}}\left\{\left|a_{i j}(t)\right|\right\}:=\bar{a}_{i j}, & \sup _{t \in \mathbb{R}}\left\{\left|b_{i j l}(t)\right|\right\}:=\bar{b}_{i j l},
\end{array}
$$

and the following conditions:

(M.3) For all $1 \leq i, j, l \leq n$,

$$
\left\{d_{i j}, a_{i j}, b_{i j l}, I_{i}\right\} \subset \mathcal{P} \mathcal{A} \mathcal{P}(\mathbb{R}, \mathbb{R}, \mu, v) .
$$

(M.4) For all $i \in\{1,2, \ldots, n\}$,

$$
\left[t \mapsto c_{i}(t)\right] \in \mathcal{A} \mathcal{P}(\mathbb{R}, \mathbb{R}) \quad \text { and } \quad \inf _{t \in \mathbb{R}}\left\{c_{i}(t)\right\}=c_{i}^{*}>0
$$


(M.5) For all $p>1$ and $1 \leq j \leq n$,

$$
f_{j}, g_{j}, h_{j} \in \mathcal{P} \mathcal{A} \mathcal{P}(\mathbb{R} \times \mathbb{R}, \mathbb{R}, \mu, v)
$$

and there exist positive continuous functions

$$
L_{j}^{f}, L_{j}^{g}, L_{j}^{h} \in L^{p}(\mathbb{R}, d \mu) \cap L^{p}(\mathbb{R}, d x)
$$

such that, for all $t, u, v \in \mathbb{R}$,

$$
\begin{aligned}
& \left|f_{j}(t, u)-f_{j}(t, v)\right|<L_{j}^{f}(t)|u-v|, \\
& \left|g_{j}(t, u)-g_{j}(t, v)\right|<L_{j}^{g}(t)|u-v|, \\
& \left|h_{j}(t, u)-h_{j}(t, v)\right|<L_{j}^{h}(t)|u-v| .
\end{aligned}
$$

In addition, we also assume that for $1 \leq j \leq n$ :

$$
f_{j}(t, 0)=g_{j}(t, 0)=h_{j}(t, 0)=0 \quad \text { for all } t \in \mathbb{R} .
$$

(M.6)

$$
q_{0}:=\max _{i \in\{1,2, \ldots, n\}}\left\{\frac{\sum_{j=1}^{n}\left[\bar{d}_{i j}\left\|L_{j}^{f}\right\|_{p}+\bar{a}_{i j}\left\|L_{j}^{g}\right\|_{p}+\sum_{l=1}^{n} \bar{b}_{i j l}\left(\left\|L_{j}^{h}\right\|_{p}\left\|h_{l}\right\|_{\infty}+\left\|L_{l}^{h}\right\|_{p}\left\|h_{j}\right\|_{\infty}\right)\right]}{\left(q c_{i}^{*}\right)^{\frac{1}{q}}}\right\}<1 .
$$

Next, define

$$
\begin{aligned}
& L:=\max _{i \in\{1,2, \ldots, n\}}\left\{\frac{\bar{I}_{i}}{c_{i}^{*}}\right\}, \\
& p_{0}:=\max _{i \in\{1,2, \ldots, n\}}\left\{\frac{\sum_{j=1}^{n}\left[\bar{d}_{i j}\left\|L_{j}^{f}\right\|_{p}+\bar{a}_{i j}\left\|L_{j}^{g}\right\|_{p}+\sum_{l=1}^{n} \bar{b}_{i j l}\left\|L_{j}^{h}\right\|_{p}\left\|h_{l}\right\|_{\infty}\right]}{\left(q c_{i}^{*}\right)^{\frac{1}{q}}}\right\} .
\end{aligned}
$$

Remark 3.1 If $q_{0}<1$, then $p_{0}<1$.

Lemma 3.2 Suppose that measures $\mu, v \in \mathcal{M}$ satisfy the following requirements:

- $p>1$ and condition (M.2) holds;

- $\Lambda \in \mathcal{C}(\mathbb{R} \times \mathbb{R}, \mathbb{R})$ is a Lipschitz function such that $L^{\Lambda} \in L^{p}(\mathbb{R}, d \mu)$; and

- $y \in \mathcal{P} \mathcal{A P}(\mathbb{R}, \mathbb{R}, \mu, v)$.

Then $[s \mapsto \Lambda(s, y(s-\theta))] \in \mathcal{P} \mathcal{A} \mathcal{P}(\mathbb{R}, \mathbb{R}, \mu, \nu)$, where $\theta \in \mathbb{R}$.

Proof Since $y \in \mathcal{P} \mathcal{A P}(\mathbb{R}, \mathbb{R}, \mu, \nu)$, it follows that

$$
y=y_{1}+y_{2}, \quad \text { where } y_{1} \in \mathcal{A P}(\mathbb{R}, \mathbb{R}) \text { and } y_{2} \in \mathcal{E}(\mathbb{R}, \mathbb{R}, \mu, v) .
$$

Let

$$
\Psi(t)=\Lambda\left(t, y_{1}(t-\theta)\right)+\left[\Lambda\left(t, y_{1}(t-\theta)+y_{2}(t-\theta)\right)-\Lambda\left(t, y_{1}(t-\theta)\right)\right]=\Psi_{1}(t)+\Psi_{2}(t)
$$


where

$$
\Psi_{1}(t)=\Lambda\left(t, y_{1}(t-\theta)\right) \quad \text { and } \quad \Psi_{2}(t)=\Lambda\left(t, y_{1}(t-\theta)+y_{2}(t-\theta)\right)-\Lambda\left(t, y_{1}(t-\theta)\right)
$$

Applying [14], we can conclude that $\Psi_{1} \in \mathcal{A P}(\mathbb{R}, \mathbb{R})$.

Next, we prove that $\Psi_{2} \in \mathcal{E}(\mathbb{R}, \mathbb{R}, \mu, v)$. Let $z>0$, then we have

$$
\begin{aligned}
& \frac{1}{v([-z, z])} \int_{-z}^{z}\left|\Psi_{2}(t)\right| d \mu(t) \\
& \quad=\frac{1}{v([-z, z])} \int_{-z}^{z}\left|\Lambda\left(t, y_{1}(t-\theta)+y_{2}(t-\theta)\right)-\Lambda\left(t, y_{1}(t-\theta)\right)\right| d \mu(t) \\
& \quad \leq \frac{1}{v([-z, z])} \int_{-z}^{z} L^{\Lambda}(t)\left|y_{2}(t-\theta)\right| d \mu(t) .
\end{aligned}
$$

Since condition (M.2) holds and $y_{2} \in \mathcal{E}(\mathbb{R}, \mathbb{R}, \mu, v)$, we get

$$
\begin{aligned}
& \frac{1}{v([-z, z])} \int_{-z}^{z}\left|\Psi_{2}(t)\right| d \mu(t) \\
& \quad \leq \frac{1}{v([-z, z])} \int_{-z}^{z} L^{\Lambda}(t)\left|y_{2}(t-\theta)\right| d \mu(t) \\
& \quad \leq \frac{\left\|y_{2}\right\|_{\infty}}{v([-z, z])} \int_{-z}^{z} L^{\Lambda}(t) d \mu(t) \\
& \quad \leq \frac{\left\|y_{2}\right\|_{\infty}}{v([-z, z])}\left[\int_{-z}^{z}\left(L^{\Lambda}(t)\right)^{p} d \mu(t)\right]^{\frac{1}{p}}\left[\int_{-z}^{z} d \mu(t)\right]^{\frac{1}{q}}, \quad \text { where } \frac{1}{p}+\frac{1}{q}=1 \\
& \quad \leq \frac{\left\|y_{2}\right\|_{\infty}}{v([-z, z])^{\frac{1}{p}}}\left\|L^{\Lambda}\right\|_{p}\left[\frac{\mu([-z, z])}{v([-z, z])}\right]^{\frac{1}{q}} \rightarrow 0, \quad \text { as } z \rightarrow+\infty .
\end{aligned}
$$

Therefore

$$
\left[t \mapsto \Psi_{2}(t)\right] \in \mathcal{E}(\mathbb{R}, \mathbb{R}, \mu, v) \quad \text { and } \quad[s \mapsto \Lambda(s, y(s-\theta))] \in \mathcal{P} \mathcal{A} \mathcal{P}(\mathbb{R}, \mathbb{R}, \mu, v)
$$

This completes the proof of Lemma 3.2.

If measures $\mu$ and $v$ are equal, then hypothesis (M.2) is satisfied and we can deduce the following corollary.

Corollary 3.3 Suppose that measure $\mu \in \mathcal{M}$ satisfies the following conditions:

- $p>1$

- $\Lambda \in \mathcal{C}(\mathbb{R} \times \mathbb{R}, \mathbb{R})$ is a Lipschitz function such that $L^{\Lambda} \in L^{p}(\mathbb{R}, d \mu)$; and

- $y \in \mathcal{P} \mathcal{A} \mathcal{P}(\mathbb{R}, \mathbb{R}, \mu)$.

Then $[s \mapsto \Lambda(s, y(s-\theta))] \in \mathcal{P} \mathcal{A} \mathcal{P}(\mathbb{R}, \mathbb{R}, \mu)$, where $\theta \in \mathbb{R}$.

Lemma 3.4 Let $\mu, v \in \mathcal{M}$ and suppose that 
Then

$$
y \times z \in \mathcal{P} \mathcal{A} \mathcal{P}(\mathbb{R}, \mathbb{R}, \mu, v) .
$$

Proof Since $y, z \in \mathcal{P} \mathcal{A P}(\mathbb{R}, \mathbb{R}, \mu, v)$, it follows that

$$
y=y_{1}+y_{2} \quad \text { and } \quad z=z_{1}+z_{2}, \quad \text { where } y_{1}, z_{1} \in \mathcal{A P}(\mathbb{R}, \mathbb{R}) \text { and } y_{2}, z_{2} \in \mathcal{E}(\mathbb{R}, \mathbb{R}, \mu, v) \text {. }
$$

Then

$$
y \times z=y_{1} z_{1}+y_{2} z_{1}+y_{1} z_{2}+y_{2} z_{2} .
$$

We shall show that $y_{1} z_{1} \in \mathcal{A P}(\mathbb{R}, \mathbb{R})$. Letting $\varphi_{0} \in \mathcal{A P}(\mathbb{R}, \mathbb{R})$, we see that

$$
\left\|\varphi_{0}^{2}(t)-\varphi_{0}^{2}(t+\tau)\right\|=\left\|\varphi_{0}(t)+\varphi_{0}(t+\tau)\right\| \cdot\left\|\varphi_{0}(t)-\varphi_{0}(t+\tau)\right\| \leq 2\left\|\varphi_{0}\right\|_{\infty} \cdot \varepsilon .
$$

Then $\varphi_{0}^{2} \in \mathcal{A P}(\mathbb{R}, \mathbb{R})$, so it follows that

$$
\left(y_{1}+z_{1}\right)^{2} \in \mathcal{A P}(\mathbb{R}, \mathbb{R}) \quad \text { and } \quad\left(y_{1}-z_{1}\right)^{2} \in \mathcal{A P}(\mathbb{R}, \mathbb{R})
$$

since

$$
\left(y_{1}+z_{1}\right) \in \mathcal{A P}(\mathbb{R}, \mathbb{R}) \quad \text { and } \quad\left(y_{1}-z_{1}\right) \in \mathcal{A} \mathcal{P}(\mathbb{R}, \mathbb{R}) .
$$

Note that

$$
y_{1} \times z_{1}=\frac{1}{4}\left(\left(y_{1}+z_{1}\right)^{2}-\left(y_{1}-z_{1}\right)^{2}\right),
$$

so we can conclude that indeed $y_{1} z_{1} \in \mathcal{A P}(\mathbb{R}, \mathbb{R})$.

Next, we shall prove that

$$
y_{2} z_{1}+y_{1} z_{2}+y_{2} z_{2} \in \mathcal{E}(\mathbb{R}, \mathbb{R}, \mu, \nu)
$$

Indeed, for $z>0$, we have

$$
\begin{aligned}
& \frac{1}{v([-z, z])} \int_{-z}^{z}\left|\left(y_{1} z_{2}+y_{2} z_{1}+y_{2} z_{2}\right)(t)\right| d \mu(t) \\
& \leq \frac{\left\|y_{1}\right\|_{\infty}}{v([-z, z])} \int_{-z}^{z}\left|z_{2}(t)\right| d \mu(t)+\frac{\left\|z_{1}\right\|_{\infty}}{v([-z, z])} \int_{-z}^{z}\left|y_{2}(t)\right| d \mu(t) \\
& \quad+\frac{\left\|y_{2}\right\|_{\infty}}{v([-z, z])} \int_{-z}^{z}\left|z_{2}(t)\right| d \mu(t) .
\end{aligned}
$$

Since $y_{2}, z_{2} \in \mathcal{E}(\mathbb{R}, \mathbb{R}, \mu, \nu)$, this completes the proof of Lemma 3.4.

Next, we define the nonlinear operator $\Gamma$ as follows: for any $\varphi=\left(\varphi_{1}, \ldots, \varphi_{n}\right) \in$ $\mathcal{P} \mathcal{A} \mathcal{P}\left(\mathbb{R}, \mathbb{R}^{n}, \mu, v\right)$

$$
(\Gamma \circ \varphi)(t):=x_{\varphi}(t)=\left(\int_{-\infty}^{t} F_{1}(s) e^{-\int_{s}^{t} c_{1}(u) d u} d s, \ldots, \int_{-\infty}^{t} F_{n}(s) e^{-\int_{s}^{t} c_{n}(u) d u} d s\right)^{T}
$$


and

$$
\begin{aligned}
F_{i}(s)= & \sum_{j=1}^{n} d_{i j}(s) f_{j}\left(s, \varphi_{j}(s)\right)+\sum_{j=1}^{n} a_{i j}(s) g_{j}\left(s, \varphi_{j}\left(s-\tau_{i j}\right)\right) \\
& +\sum_{j=1}^{n} \sum_{l=1}^{n} b_{i j l}(s) h_{j}\left(s, \varphi_{j}\left(s-\sigma_{i j}\right)\right) h_{l}\left(s, s-v_{i j}\right)+I_{i}(s) .
\end{aligned}
$$

Lemma 3.5 Suppose that conditions (M.1)-(M.6) hold. Then $\Gamma$ maps $\mathcal{P} \mathcal{A} \mathcal{P}\left(\mathbb{R}, \mathbb{R}^{n}, \mu, v\right)$ into itself.

Proof Let $\varphi=\left(\varphi_{1}, \ldots, \varphi_{n}\right) \in \mathcal{P} \mathcal{A} \mathcal{P}\left(\mathbb{R}, \mathbb{R}^{n}, \mu, \nu\right)$. Then the function

$$
\begin{aligned}
F_{i}: s \mapsto & \sum_{i=1}^{n} d_{i j}(s) f_{j}\left(s, \varphi_{j}(s)\right)+\sum_{j=1}^{n} a_{i j}(s) g_{j}\left(s, \varphi_{j}\left(s-\tau_{i j}\right)\right) \\
& +\sum_{j=1}^{n} \sum_{l=1}^{n} b_{i j l}(s) h_{j}\left(s, s-\sigma_{i j}\right) h_{l}\left(\varphi_{l}\left(s, s-v_{i j}\right)\right)+I_{i}(s)
\end{aligned}
$$

is double measure pseudo almost periodic for all $1 \leq i \leq n$, by Lemmas 2.2, 3.2, and 3.4.

Hence, for all $1 \leq i \leq n$, we have

$$
F_{i}=F_{i}^{1}+F_{i}^{2}, \quad \text { where } F_{i}^{1} \in \mathcal{A P}(\mathbb{R}, \mathbb{R}) \text { and } F_{i}^{2} \in \mathcal{E}(\mathbb{R}, \mathbb{R}, \mu, v)
$$

Therefore

$$
\begin{aligned}
\left(\Gamma_{i} \circ \varphi\right)(t) & =\int_{-\infty}^{t} e^{-\int_{s}^{t} c_{i}(u) d u} F_{i}^{1}(s) d s+\int_{-\infty}^{t} e^{-\int_{s}^{t} c_{i}(u) d u} F_{i}^{2}(s) d s \\
& =\left(\Gamma_{i} \circ F_{i}^{1}\right)(t)+\left(\Gamma_{i} \circ F_{i}^{2}\right)(t) .
\end{aligned}
$$

We have to prove that $\Gamma_{i} \circ F_{i}^{1} \in \mathcal{A P}(\mathbb{R}, \mathbb{R}), i \in\{1,2,3, \ldots, n\}$. To this end, note that

$$
\begin{aligned}
\left|\left(\Gamma_{i} \circ F_{i}^{1}\right)(t+\tau)-\left(\Gamma_{i} \circ F_{i}^{1}\right)(t)\right| & =\left|\int_{-\infty}^{t+\tau} e^{-\int_{s}^{t+\tau} c_{i}(u) d u} F_{i}^{1}(s) d s-\int_{-\infty}^{t} e^{-\int_{s}^{t} c_{i}(u) d u} F_{i}^{1}(s) d s\right| \\
& \leq\left|\int_{0}^{+\infty} e^{-y c_{i}^{*}} F_{i}^{1}(t+\tau-y) d y-\int_{0}^{+\infty} e^{-y c_{i}^{*}} F_{i}^{1}(t-y) d y\right| \\
& \leq \int_{0}^{+\infty} e^{-y c_{i}^{*}}\left|F_{i}^{1}(t+\tau-y)-F_{i}^{1}(t-y)\right| d y \\
& \leq \varepsilon \int_{0}^{+\infty} e^{-y c_{i}^{*}} d y=\frac{\varepsilon}{c_{i}^{*}} .
\end{aligned}
$$

Therefore $\Gamma_{i} \circ F_{i}^{1} \in \mathcal{A P}\left(\mathbb{R}, \mathbb{R}^{n}\right), i \in\{1,2,3, \ldots, n\}$.

On the other hand, we can prove that $\Gamma_{i} \circ F_{i}^{2} \in \mathcal{E}(\mathbb{R}, \mathbb{R}, \mu, v)$ for $i \in\{1,2,3, \ldots, n\}$. To this end, note that

$$
\int_{-z}^{z}\left|\left(\Gamma_{i} \circ F_{i}^{2}\right)(t)\right| d \mu(t)=\int_{-z}^{z}\left|\int_{-\infty}^{t} e^{-\int_{s}^{t} c_{i}(u) d u} F_{i}^{2}(s) d s\right| d \mu(t) .
$$


Using Fubini's theorem, we get

$$
\begin{aligned}
\frac{1}{v([-z, z])} \int_{-z}^{z}\left|\left(\Gamma_{i} \circ F_{i}^{2}\right)(t)\right| d \mu(t) & =\frac{1}{v([-z, z])} \int_{-z}^{z}\left|\int_{-\infty}^{t} e^{-\int_{s}^{t} c_{i}(u) d u} F_{i}^{2}(s) d s\right| d \mu(t) \\
& \leq \frac{1}{v([-z, z])} \int_{-z}^{z} \int_{0}^{\infty} e^{-y c_{i}^{*}}\left|F_{i}^{2}(t-y)\right| d s d \mu(t) \\
& \leq \frac{1}{v([-z, z])} \int_{0}^{\infty} \int_{-z}^{z} e^{-y c_{i}^{*}}\left|F_{i}^{2}(t-y)\right| d s d \mu(t)
\end{aligned}
$$

for all $z>0$. Since $F_{i}^{2} \in \mathcal{E}(\mathbb{R}, \mathbb{R}, \mu, v)$, it follows by Lemma 2.2 and the dominated convergence theorem that

$$
\Gamma_{i} \circ F_{i}^{2} \in \mathcal{E}(\mathbb{R}, \mathbb{R}, \mu, v) \quad \text { for all } i \in\{1,2,3, \ldots, n\}
$$

We can thus conclude that

$$
\Gamma_{i} \circ \varphi \in \mathcal{P} \mathcal{A P}(\mathbb{R}, \mathbb{R}, \mu, \nu) \text { for all } i \in\{1,2,3, \ldots, n\}
$$

hence

$$
\Gamma \circ \varphi \in \mathcal{P} \mathcal{A P}\left(\mathbb{R}, \mathbb{R}^{n}, \mu, v\right)
$$

This completes the proof of Lemma 3.5.

Theorem 3.6 Suppose that conditions (M.1)-(M.6) hold. Then system (1.1) admits a unique $(\mu, v)$-pap solution in $\mathbb{E}$, where

$$
\mathbb{E}=\left\{\psi \in \mathcal{P} \mathcal{A} \mathcal{P}\left(\mathbb{R}, \mathbb{R}^{n}, \mu, \nu\right):\left\|\psi-\varphi_{0}\right\|_{\infty} \leq \frac{p_{0} L}{1-p_{0}}\right\}
$$

and

$$
\varphi_{0}(t)=\left(\int_{-\infty}^{t} e^{-\int_{s}^{t} c_{1}(u) d u} I_{1}(s) d s, \ldots, \int_{-\infty}^{t} e^{-\int_{s}^{t} c_{n}(u) d u} I_{n}(s) d s\right)^{T}
$$

Proof We have

$$
\left\|\varphi_{0}\right\|_{\infty}=\max _{i \in\{1,2, \ldots, n\}} \sup _{t \in \mathbb{R}}\left(\left|\int_{-\infty}^{t} e^{-\int_{s}^{t} c_{i}(u) d u} I_{i}(s) d s\right|\right) \leq \max _{i \in\{1,2, \ldots, n\}}\left(\frac{\bar{I}_{i}}{c_{i}^{*}}\right):=L
$$

and

$$
\|\varphi\|_{\infty} \leq\left\|\varphi-\varphi_{0}\right\|_{\infty}+\|\varphi\|_{\infty} \leq\left\|\varphi-\varphi_{0}\right\|_{\infty}+L \leq \frac{L}{1-p_{0}} .
$$

Let

$$
\mathbb{E}=\mathbb{E}\left(\varphi_{0}, p_{0}\right)=\left\{\varphi \in \mathcal{P} \mathcal{A} \mathcal{P}\left(\mathbb{R}, \mathbb{R}^{n}, \mu, \nu\right):\left\|\varphi-\varphi_{0}\right\|_{\infty} \leq \frac{p_{0} L}{1-p_{0}}\right\}
$$


Then, for every $\varphi \in \mathbb{E}$, we obtain the following:

$$
\begin{aligned}
& \left\|(\Gamma \circ \varphi)-\varphi_{0}\right\|_{\infty}=\max _{i \in\{1,2, \ldots, n\}} \sup _{t \in \mathbb{R}}\left\{\mid \int_{-\infty}^{t} e^{-\int_{s}^{t} c_{i}(u) d u}\right. \\
& \times \sum_{j=1}^{n}\left[d_{i j}(s) f_{j}\left(s, \varphi_{j}(s)\right)+a_{i j}(s) g_{j}\left(s, \varphi_{j}\left(s-\tau_{i j}\right)\right)\right. \\
& \left.\left.+\sum_{l=1}^{n} b_{i j l}(s) h_{j}\left(s, \varphi_{j}\left(s-\sigma_{i j}\right)\right) h_{l}\left(s, \varphi_{l}\left(s-v_{i j}\right)\right)\right] d s \mid\right\} \\
& \leq \max _{i \in\{1,2, \ldots, n\}} \sup _{t \in \mathbb{R}}\left\{\int _ { - \infty } ^ { t } e ^ { - \int _ { s } ^ { t } c _ { i } ( u ) d u } \sum _ { j = 1 } ^ { n } \left[\bar{d}_{i j} L_{j}^{f}(s)\|\varphi\|_{\infty}+\bar{a}_{i j} L_{j}^{g}(s)\|\varphi\|_{\infty}\right.\right. \\
& \left.\left.+\sum_{l=1}^{n} \bar{b}_{i j l} L_{j}^{h}(s)\left\|h_{l}\right\|_{\infty}\|\varphi\|_{\infty}\right] d s\right\} \\
& \leq \max _{i \in\{1,2, \ldots, n\}}\left\{\frac{\sum_{j=1}^{n}\left[\bar{d}_{i j}\left\|L_{j}^{f}\right\|_{p}+\bar{a}_{i j}\left\|L_{j}^{g}\right\|_{p}+\sum_{l=1}^{n} \bar{b}_{i j l}\left\|L_{j}^{h}\right\|_{p}\left\|h_{l}\right\|_{\infty}\right]}{\left(q c_{i}^{*}\right)^{\frac{1}{q}}}\right\}\|\varphi\|_{\infty} \\
& \leq p_{0}\|\varphi\|_{\infty} \leq p_{0}\left(\left\|\varphi-\varphi_{0}\right\|_{\infty}+\left\|\varphi_{0}\right\|_{\infty}\right) \leq \frac{p_{0} L}{1-p_{0}}
\end{aligned}
$$

where

$$
p_{0}=\max _{i \in\{1,2, \ldots, n\}}\left\{\frac{\sum_{j=1}^{n}\left[\bar{d}_{i j}\left\|L_{j}^{f}\right\|_{\infty}+\bar{a}_{i j}\left\|L_{j}^{g}\right\|_{\infty}+\sum_{l=1}^{n} \bar{b}_{i j l}\left\|L_{j}^{h}\right\|_{\infty}\left\|h_{l}\right\|_{\infty}\right]}{\left(q c_{i}^{*}\right)^{\frac{1}{q}}}\right\}<1 .
$$

Therefore $\Gamma \circ \varphi \in \mathbb{E}$.

Next, for all $\phi, \psi \in \mathbb{E}$, we get the following:

$$
\begin{aligned}
& \left|\left(\Gamma_{i} \circ \phi\right)(t)-\left(\Gamma_{i} \circ \psi\right)(t)\right| \\
& \leq \int_{-\infty}^{t} e^{-\int_{s}^{t} c_{i}(u) d u} \sum_{j=1}^{n} \mid d_{i j}(s)\left(f_{j}\left(s, \phi_{j}(s)\right)-f_{j}\left(s, \psi_{j}(s)\right)\right) \\
& +a_{i j}(s)\left(g_{j}\left(s, \phi_{j}\left(s-\tau_{i j}\right)\right)-g_{j}\left(s, \psi_{j}\left(s-\tau_{i j}\right)\right)\right) \\
& +\sum_{l=1}^{n} b_{i j l}(s)\left(h_{j}\left(s, \phi_{j}\left(s-\sigma_{i j}\right)\right) h_{l}\left(s, \phi_{l}\left(s-v_{i j}\right)\right)\right. \\
& \left.-h_{j}\left(s, \psi_{j}\left(s-\sigma_{i j}\right)\right) h_{l}\left(s, \psi_{l}\left(s-v_{i j}\right)\right)\right) \mid d s \\
& \leq \int_{-\infty}^{t} e^{-\int_{s}^{t} c_{i}(u) d u} \sum_{j=1}^{n}\left[\bar{d}_{i j} L_{j}^{f}(s) \sup _{t \in \mathbb{R}}\left|\phi_{j}(t)-\psi_{j}(t)\right|+\bar{a}_{i j} L_{j}^{g}(s) \sup _{t \in \mathbb{R}}\left|\phi_{j}(t)-\psi_{j}(t)\right|\right. \\
& +\sum_{l=1}^{n} b_{i j l}(s) \mid h_{j}\left(s, \phi_{j}\left(s-\sigma_{i j}\right)\right) h_{l}\left(s, \phi_{l}\left(s-v_{i j}\right)\right)-h_{j}\left(s, \psi_{j}\left(s-\sigma_{i j}\right)\right) h_{l}\left(s, \phi_{l}\left(s-v_{i j}\right)\right) \\
& \left.+h_{j}\left(s, \psi_{j}\left(s-\sigma_{i j}(s)\right)\right) h_{l}\left(s, \phi_{l}\left(s-v_{i j}\right)\right)-h_{j}\left(s, \psi_{j}\left(s-\sigma_{i j}\right)\right) h_{l}\left(s, \psi_{l}\left(s-v_{i j}\right)\right) \mid\right] d s
\end{aligned}
$$




$$
\begin{aligned}
\leq & \int_{0}^{+\infty} e^{-c_{i}^{*} y} d y \sum_{j=1}^{n}\left[\bar{d}_{i j} L_{j}^{f}(s) \sup _{t \in \mathbb{R}}\left|\phi_{j}(t)-\psi_{j}(t)\right|+\bar{a}_{i j} L_{j}^{g}(s) \sup _{t \in \mathbb{R}}\left|\phi_{j}(t)-\psi_{j}(t)\right|\right. \\
& \left.+\sum_{l=1}^{n} \bar{b}_{i j l}(s)\left(L_{j}^{h}(s)\left\|h_{l}\right\|_{\infty}+L_{l}^{h}(s)\left\|h_{j}\right\|_{\infty}\right) \sup _{t \in \mathbb{R}}\left|\phi_{j}(t)-\psi_{j}(t)\right|\right] d s \\
\leq & \frac{\sum_{j=1}^{n}\left[\bar{d}_{i j}\left\|L_{j}^{f}\right\|_{p}+\bar{a}_{i j}\left\|L_{j}^{g}\right\|_{p}+\sum_{l=1}^{n} \bar{b}_{i j l}\left(\left\|L_{j}^{h}\right\|_{p}\left\|h_{l}\right\|_{\infty}+\left\|L_{l}^{h}\right\|_{p}\left\|h_{j}\right\|_{\infty}\right)\right]}{\left(q c_{i}^{*}\right)^{\frac{1}{q}}}\|\phi-\psi\|_{\infty} \\
\leq & q_{0}\|\phi-\psi\|_{\infty},
\end{aligned}
$$

where $i=1, \ldots, n$. Therefore $\|(\Gamma \circ \phi)-(\Gamma \circ \psi)\|_{\infty} \leq q_{0}\|\phi-\psi\|_{\infty}$.

Note that since $q_{0}<1, \Gamma$ is a contraction and possesses a unique fixed point $z$, which is a $(\mu, v)$-pap solution of system (1.1) in the region $\mathbb{E}$. This completes the proof of Theorem 3.6.

If the two measures $\mu$ and $v$ are equal, then according to the proof of Theorem 3.6, the following corollary can be deduced.

Corollary 3.7 Suppose that conditions (M.1) and (M.3)-(M.6) hold. Then system (1.1) admits a unique $\mu$-pap solution in

$$
\mathbb{E}=\left\{\psi \in \mathcal{P} \mathcal{A} \mathcal{P}\left(\mathbb{R}, \mathbb{R}^{n}, \mu\right):\left\|\psi-\varphi_{0}\right\|_{\infty} \leq \frac{p_{0} L}{1-p_{0}}\right\}
$$

In the sequel, we shall assume that the functions $L_{j}^{f}, L_{j}^{g}$, and $L_{j}^{h}$ are constant. By analogy, we can prove the same results as above. In addition, by the following modifications of conditions (M.5) and (M.6), the exponential stability of the solution can be obtained:

(M.7) For all $1 \leq j \leq n$, there exist constants

$$
L_{j}^{f}, L_{j}^{g}, L_{j}^{h}, M_{j}^{f}, M_{j}^{g}, M_{j}^{h} \in \mathbb{R}_{+}^{*}
$$

such that, for all $t, x_{1}, x_{2} \in \mathbb{R}$,

$$
\begin{array}{ll}
\left|f_{j}\left(t, x_{1}\right)-f_{j}\left(t, x_{2}\right)\right| \leq L_{j}^{f}\left|x_{1}-x_{2}\right|, & \left|f_{j}\left(t, x_{1}\right)\right| \leq M_{j}^{f}, \\
\left|g_{j}\left(t, x_{1}\right)-g_{j}\left(t, x_{2}\right)\right| \leq L_{j}^{g}\left|x_{1}-x_{2}\right|, & \left|g_{j}\left(t, x_{1}\right)\right| \leq M_{j}^{g}, \\
\left|h_{j}\left(t, x_{1}\right)-h_{j}\left(t, x_{2}\right)\right| \leq L_{j}^{h}\left|x_{1}-x_{2}\right|, & \left|h_{j}\left(t, x_{1}\right)\right| \leq M_{j}^{h},
\end{array}
$$

and

$$
f_{j}(t, 0)=g_{j}(t, 0)=h_{j}(t, 0)=0 .
$$

(M.8) There exists a nonnegative constant $q_{1}$ such that

$$
q_{1}:=\max _{i \in\{1,2, \ldots, n\}}\left\{\frac{\sum_{j=1}^{n}\left[\bar{d}_{i j} L_{j}^{f}+\bar{a}_{i j} L_{j}^{g}+\sum_{l=1}^{n} \bar{b}_{i j l}\left(L_{j}^{h} M_{l}^{h}+M_{j}^{h} L_{l}^{h}\right)\right]}{c_{i}^{*}}\right\}<1 .
$$


We let

$$
p_{1}:=\max _{i \in\{1,2, \ldots, n\}}\left\{\frac{\sum_{j=1}^{n}\left[\bar{d}_{i j} L_{j}^{f}+\bar{a}_{i j} L_{j}^{g}+\sum_{l=1}^{n} \bar{b}_{i j l} L_{j}^{h} M_{l}^{h}\right]}{c_{i}^{*}}\right\}
$$

and

$$
\varphi_{0}(t):=\left(\int_{-\infty}^{t} e^{-\int_{s}^{t} c_{1}(u) d u} I_{1}(s) d s, \ldots, \int_{-\infty}^{t} e^{-\int_{s}^{t} c_{n}(u) d u} I_{n}(s) d s\right)^{T} .
$$

Theorem 3.8 Suppose that conditions (M.1)-(M.4) and (M.7)-(M.8) hold. Then system (1.1) admits a unique $(\mu, \nu)$-pap solution in $\mathbb{F}$, where

$$
\mathbb{F}=\left\{\psi \in \mathcal{P} \mathcal{A} \mathcal{P}\left(\mathbb{R}, \mathbb{R}^{n}, \mu, v\right):\left\|\psi-\varphi_{0}\right\|_{\infty} \leq \frac{p_{1} L}{1-p_{1}}\right\} .
$$

Proof The following inequality holds:

$$
\left\|(\Gamma \circ \varphi)-\varphi_{0}\right\|_{\infty} \leq \frac{p_{1} L}{1-p_{1}} .
$$

Therefore $\Gamma \circ \varphi \in \mathbb{F}$. Next, for all $\phi, \psi \in \mathbb{F}$,

$$
\|(\Gamma \circ \phi)-(\Gamma \circ \psi)\|_{\infty} \leq q_{1}\|\phi-\psi\|_{\infty} .
$$

Since $q_{1}<1$, it follows that $\Gamma$ possesses a unique fixed point $z$ which is a $(\mu, \nu)$-pap solution of system (1.1) in the region $\mathbb{F}$. This completes the proof of Theorem 3.8.

If $\mu=v$, we can deduce the following result.

Corollary 3.9 Suppose that conditions (M.1), (M.3)-(M.4), and (M.7)-(M.8) hold. Then system (1.1) admits a unique $\mu$-pap solution in

$$
\mathbb{F}=\left\{\psi \in \mathcal{P} \mathcal{A} \mathcal{P}\left(\mathbb{R}, \mathbb{R}^{n}, \mu\right):\left\|\psi-\varphi_{0}\right\|_{\infty} \leq \frac{p_{1} L}{1-p_{1}}\right\} .
$$

Theorem 3.10 Suppose that conditions (M.1)-(M.4) and (M.7)-(M.8) hold. Then system (1.1) has a unique globally exponentially stable $(\mu, \nu)$-pap solution.

Proof System (1.1) has a unique $(\mu, \nu)$-pap solution

$$
z(t)=\left(z_{1}(t), \ldots, z_{n}(t)\right)^{T} \in \mathbb{E}
$$

and $u(t)=\left(u_{1}(t), \ldots, u_{n}(t)\right)^{T}$ is the initial value.

Let $x(t)=\left(x_{1}(t), \ldots, x_{n}(t)\right)^{T}$ be an arbitrary solution of system (1.1) with initial value $\varphi^{*}(t)=\left(\varphi_{1}^{*}(t), \ldots, \varphi_{n}^{*}(t)\right)^{T}$. Let $y_{i}(t)=x_{i}(t)-z_{i}(t), \varphi_{i}(t)=\varphi_{i}^{*}(t)-u_{i}(t)$ for $i=1, \ldots, n$. 
Then

$$
\begin{aligned}
y_{i}^{\prime}(t)= & -c_{i}(t) y_{i}(t) \\
& +\sum_{j=1}^{n}\left(d_{i j}(t)\left(f_{j}\left(t, x_{j}(t)\right)-f_{j}\left(t, z_{j}(t)\right)\right)\right. \\
& +a_{i j}(t)\left[g_{j}\left(t, x_{j}\left(t-\tau_{i j}\right)\right)-g_{j}\left(t, z_{j}\left(t-\tau_{i j}\right)\right)\right] \\
& +\sum_{l=1}^{n} b_{i j l}(t)\left[h _ { j } \left(t, x_{j}\left(t-\sigma_{i j}\right) h_{l}\left(t, x_{l}\left(t-v_{i j}\right)\right)\right.\right. \\
& \left.\left.-h_{j}\left(t, z_{j}\left(t-\sigma_{i j}\right)\right) h_{l}\left(t, z_{l}\left(t-v_{i j}\right)\right)\right]\right),
\end{aligned}
$$

where $i \in\{1,2,3, \ldots, n\}$. Let $F_{i}$ be defined by

$$
F_{i}(w)=c_{i}^{*}-w-\sum_{j=1}^{n}\left[\bar{d}_{i j} L_{j}^{f}+\bar{a}_{i j} L_{j}^{g} e^{w \tau_{i j}}+\sum_{l=1}^{n} \bar{b}_{i j l}\left(L_{j}^{h} e^{w \sigma_{i j}} M_{l}^{h}+M_{j}^{h} L_{l}^{h} e^{w v_{i j}}\right)\right] .
$$

By condition (M.8), we have

$$
F_{i}(0)=c_{i}^{*}-\sum_{j=1}^{n}\left[\bar{d}_{i j} L_{j}^{f}+\bar{a}_{i j} L_{j}^{g}+\sum_{l=1}^{n} \bar{b}_{i j l}\left(L_{j}^{h} M_{l}^{h}+M_{j}^{h} L_{l}^{h}\right)\right]>0 .
$$

Thus there exists $\varepsilon_{i}^{*}>0$ such that $F_{i}\left(\varepsilon_{i}^{*}\right)=0$ and $F_{i}\left(\varepsilon_{i}>0\right)$ if $\varepsilon_{i} \in\left(0, \varepsilon_{i}^{*}\right)$.

Let $\eta=\min \left\{\varepsilon_{1}^{*}, \ldots, \varepsilon_{n}^{*}\right\}$. Then $F_{i}(\eta) \geq 0$ if $i=1, \ldots, n$. Next, there exists a nonnegative $\lambda$ such that

$$
0<\lambda<\min \left\{\eta, c_{1}^{*}, \ldots, c_{n}^{*}\right\} \quad \text { and } \quad F_{i}(\lambda)>0 \text {, }
$$

so for all $i \in\{1, \ldots, n\}$,

$$
\frac{1}{c_{i}^{*}-\lambda}\left[\sum_{j=1}^{n}\left(\bar{c}_{i j} L_{j}^{f}+\bar{d}_{i j} L_{j}^{g} e^{\lambda \tau_{i j}}\right)+\sum_{j=1}^{n} \sum_{l=1}^{n} \bar{b}_{i j l}\left(L_{j}^{h} e^{\lambda \sigma_{i j}} M_{l}^{h}+M_{j}^{h} L_{l}^{h} e^{\lambda v_{i j}}\right)\right]<1 .
$$

Multiplying (3.3)-(3.5) by $e^{\int_{0}^{s} c_{i}(u) d u}$ and integrating on $[0, t]$, we get

$$
\begin{aligned}
y_{i}(t)= & \varphi_{i}(0) e^{-\int_{0}^{t} c_{i}(u) d u}+\int_{0}^{t} e^{-\int_{s}^{t} c_{i}(u) d u} \sum_{j=1}^{n}\left(d_{i j}(s)\left[f_{j}\left(s, y_{j}(s)+z_{j}(s)\right)-f_{j}\left(s, z_{j}(s)\right)\right]\right. \\
& +a_{i j}(s)\left[g_{j}\left(s, y_{j}\left(s-\tau_{i j}\right)+z_{j}\left(s-\tau_{i j}\right)\right)-g_{j}\left(s, z_{j}\left(s-\tau_{i j}\right)\right)\right] \\
& +\sum_{l=1}^{n} b_{i j l}(s)\left[h_{j}\left(s, y_{j}\left(s-\sigma_{i j}\right)+z_{j}\left(s-\sigma_{i j}\right)\right) h_{l}\left(s, y_{j}\left(t-v_{i j}\right)+z_{j}\left(t-v_{i j}\right)\right)\right. \\
& \left.\left.-h_{j}\left(z_{j}\left(t-\sigma_{i j}\right)\right) h_{l}\left(z_{l}\left(t-v_{i j}\right)\right)\right]\right) d s .
\end{aligned}
$$

Let

$$
M=\max _{1 \leq i \leq n} \frac{c_{i}^{*}}{\sum_{j=1}^{n}\left[\left(\overline{(}_{i j} L_{j}^{f}+\bar{a}_{i j} L_{j}^{g}\right)+\sum_{l=1}^{n} \bar{b}_{i j l}\left(L_{j}^{h} M_{l}^{h}+M_{j}^{h} L_{l}^{h}\right)\right]} .
$$


Clearly, $M>1$, and

$$
\frac{1}{M}-\frac{1}{c_{i}^{*}-\lambda}\left[\sum_{j=1}^{n}\left(\bar{c}_{i j} L_{j}^{f}+\bar{d}_{i j} L_{j}^{g} e^{\lambda \tau_{i j}}\right)+\sum_{j=1}^{n} \sum_{l=1}^{n} \bar{b}_{i j l}\left(L_{j}^{h} e^{\lambda \sigma_{i j}} M_{l}^{h}+M_{j}^{h} L_{l}^{h} e^{\lambda v_{i j}}\right)\right] \leq 0
$$

where $0<\lambda<\min \left\{\eta, c_{1}^{*}, c_{2}^{*}, \ldots, c_{n}^{*}\right\}$ is as in (3.6). Also,

$$
\|y(t)\|_{\infty} \leq M\|\varphi\|_{\infty} e^{-\lambda t}, \quad t>0 .
$$

To prove inequality (3.7), we first show that, for any $u>1$, the following inequality holds:

$$
\|y(t)\|_{\infty}<u M\|\varphi\|_{\infty} e^{-\lambda t}, \quad t>0
$$

Indeed, if (3.8) were false, there would exist some $t_{1}>0$ and $i \in\{1, \ldots, n\}$ such that

$$
\left\|y\left(t_{1}\right)\right\|_{\infty}=\left\|y_{i}\left(t_{1}\right)\right\|_{\infty}=u M\|\varphi\|_{\infty} e^{-\lambda t_{1}}
$$

and

$$
\|y(t)\|_{\infty} \leq u M\|\varphi\|_{\infty} e^{-\lambda t} \quad \text { for every } t \in\left(-\infty, t_{1}\right]
$$

So we could obtain

$$
\begin{aligned}
& \left|y\left(t_{1}\right)\right| \leq\|\varphi\|_{\infty} e^{-t_{1} c_{i}^{*}}+\int_{0}^{t_{1}} e^{\left(-t_{1}-s\right) c_{i}^{*}} \sum_{j=1}^{n}\left[\bar{d}_{i j} L_{j}^{f}\left\|y_{j}(s)\right\|_{\infty}+\bar{a}_{i j} L_{j}^{g}\left\|y_{j}\left(s-\tau_{i j}\right)\right\|_{\infty}\right. \\
& \left.+\sum_{l=1}^{n} \bar{b}_{i j l}\left(L_{j}^{h} M_{l}^{h}\left\|y_{j}\left(s-\sigma_{i j}\right)\right\|_{\infty}+M_{j}^{h} L_{l}^{h}\left\|y_{j}\left(s-v_{i j}\right)\right\|_{\infty}\right)\right] d s \\
& \leq\|\varphi\|_{\infty} e^{-t_{1} c_{i}^{*}}+\int_{0}^{t_{1}} e^{-\left(t_{1}-s\right) c_{i}^{*}} u M \sum_{j=1}^{n}\left[\bar{d}_{i j} L_{j}^{f}\|\varphi\|_{\infty} e^{-\lambda s}+\bar{a}_{i j} L_{j}^{g}\|\varphi\|_{\infty} e^{-\lambda\left(s-\tau_{i j}\right)}\right. \\
& \left.+\sum_{l=1}^{n} \bar{b}_{i j l}\left(L_{j}^{h} M_{l}^{h}\|\varphi\|_{\infty} e^{-\lambda\left(s-\sigma_{i j}\right)}+M_{j}^{h} L_{l}^{h}\|\varphi\|_{\infty} e^{-\lambda\left(s-v_{i j}\right)}\right)\right] d s \\
& \leq\|\varphi\|_{\infty} e^{-t_{1} c_{i}^{*}}+\int_{0}^{t_{1}} e^{-\left(t_{1}-s\right) c_{i}^{*}} u M\|\varphi\|_{\infty} e^{-\lambda s} \sum_{j=1}^{n}\left[\bar{d}_{i j} L_{j}^{f}+\bar{a}_{i j} L_{j}^{g} e^{\lambda \tau_{i j}}\right. \\
& \left.+\sum_{l=1}^{n} \bar{b}_{i j l}\left(L_{j}^{h} M_{l}^{h} e^{\lambda \sigma_{i j}}+M_{j}^{h} L_{l}^{h} e^{\lambda v_{i j}}\right)\right] d s \\
& \leq u M\|\varphi\|_{\infty} e^{-\lambda t_{1}}\left[e ^ { ( \lambda - a _ { i * } ) t _ { 1 } } \left(\frac{1}{M}-\frac{1}{c_{i}^{*}-\lambda}\left[\sum _ { j = 1 } ^ { n } \left\{\bar{c}_{i j} L_{j}^{f}+\bar{d}_{i j} L_{j}^{g} e^{\lambda \tau_{i j}}\right.\right.\right.\right. \\
& \left.\left.\left.+\sum_{l=1}^{n} \bar{b}_{i j l}\left(L_{j}^{h} e^{\lambda \sigma_{i j}} M_{l}^{h}+M_{j}^{h} L_{l}^{h} e^{\lambda v_{i j}}\right)\right\}\right]\right) \\
& \left.+\frac{1}{c_{i}^{*}-\lambda}\left[\sum_{j=1}^{n}\left(\bar{d}_{i j} L_{j}^{f}+\bar{a}_{i j} L_{j}^{g} e^{\lambda \tau_{i j}}\right)+\sum_{j=1}^{n} \sum_{l=1}^{n} \bar{b}_{i j l}\left(L_{j}^{h} e^{\lambda \sigma_{i j}} M_{l}^{h}+M_{j}^{h} L_{l}^{h} e^{\lambda v_{i j}}\right)\right]\right]
\end{aligned}
$$




$$
\begin{aligned}
\leq & u M\|\varphi\|_{\infty} e^{-\lambda t_{1}} \frac{1}{c_{i}^{*}-\lambda}\left[\sum _ { j = 1 } ^ { n } \left(\left(\bar{c}_{i j} L_{j}^{f}+\bar{d}_{i j} L_{j}^{g} e^{\lambda \tau_{i j}}\right)\right.\right. \\
& \left.\left.+\sum_{l=1}^{n} \bar{b}_{i j l}\left(L_{j}^{h} e^{\lambda \sigma_{i j}} M_{l}^{h}+M_{j}^{h} L_{l}^{h} e^{\lambda v_{i j}}\right)\right)\right] \\
= & u M\|\varphi\|_{\infty} e^{-\lambda t_{1}} .
\end{aligned}
$$

Hence we could conclude that $\left\|y\left(t_{1}\right)\right\|_{\infty}<u M\|\varphi\|_{\infty} e^{-\lambda t_{1}}$, which contradicts inequality (3.8). Note that $u \rightarrow 1$, so (3.7) holds. Therefore system (1.1) has a unique globally exponentially stable $(\mu, v)$-pap solution. This completes the proof of Theorem 3.10.

If $\mu=v$, then hypothesis (M.2) is satisfied, and we can deduce the following corollary:

Corollary 3.11 Suppose that conditions (M.1), (M.3)-(M.4), and (M.7)-(M.8) hold. Then system (1.1) has a unique globally exponentially stable $\mu$-pap solution.

\section{An application to neural networks}

Neural networks have attracted a lot of attention in recent years, and especially the special case of the so-called high-order Hopfield neural networks (HOHNNs), which have been intensively investigated by many scholars in recent years because of their stronger approximation characteristics, larger storage capacity, faster convergence speed, and higher fault tolerance than low-order Hopfield neural networks. Many excellent results about their dynamic characteristics have been obtained, e.g., [2-4, 7, 14, 22, 24, 25]. Clearly, the study of the oscillations and dynamics of such models is an exciting new topic.

Using the results from this paper, we prove the existence, the exponential stability, and the uniqueness of $(\mu, v)$-pap solutions of the following models of high-order Hopfield neural networks (HOHNNs) with delays:

$$
\begin{aligned}
x_{i}^{\prime}(t)= & -c_{i}(t) x_{i}(t)+\sum_{j=1}^{n} d_{i j}(t) f_{j}\left(t, x_{j}(t)\right)+\sum_{j=1}^{n} a_{i j}(t) g_{j}\left(t, x_{j}\left(t-\tau_{i j}\right)\right) \\
& +\sum_{j=1}^{n} \sum_{l=1}^{n} b_{i j l}(t) h_{j}\left(t, x_{j}\left(t-\sigma_{i j}\right)\right) h_{l}\left(t, x_{l}\left(t-v_{i j}\right)\right)+I_{i}(t),
\end{aligned}
$$

where $i \in\{1, \ldots, n\}$.

- $n-$ number of neurons in neural network;

- $x_{i}(t)$ - ith neuron at time $t$

- $f_{j}, g_{j}, h_{j}$-activation function of $j$ th neuron;

- $d_{i j}(t), a_{i j}(t), b_{i j l}(t)$-functions connection weights;

- $I_{i}(t)$-external inputs at time $t$;

- $c_{i}(t)>0$-rate of $i$ th neuron;

- $\tau_{i j} \geq 0, \sigma_{i j} \geq 0, v_{i j} \geq 0$-transmission delays.

The initial conditions associated with system (4.1) are of the form

$$
x_{i}(s)=\varphi_{i}(s), \quad s \in(-\theta, 0], \quad i=1,2, \ldots, n .
$$

In our paper we have generalized the previous results by using the notion of double measure and working with two-variable functions. 
Example 4.1 Consider the following model:

$$
\begin{aligned}
x_{i}^{\prime}(t)= & -c_{i}(t) x_{i}(t)+\sum_{j=1}^{2} d_{i j}(t) f_{j}\left(t, x_{j}(t)\right)+\sum_{j=1}^{2} a_{i j}(t) g_{j}\left(t, x_{j}(t-1)\right) \\
& +\sum_{j=1}^{2} \sum_{l=1}^{2} b_{i j l}(t) h_{j}\left(t, x_{j}(t-1)\right) h_{l}\left(t, x_{l}(t-1)\right)+I_{i}(t), \quad 1 \leq i \leq 2,
\end{aligned}
$$

where $c_{1}=c_{2}=2, g_{1}(t)=g_{2}(t)=\sin t$. Then

$$
L^{g_{1}}=L^{g_{2}}=M^{g_{1}}=M^{g_{2}}=1, \quad \tau_{i j}=\sigma_{i j}=v_{i j}=1 .
$$

Measures $\mu$ and $v$ are defined by the following double weights, respectively:

$$
\rho_{1}(t)=e^{\sin (t)}, \quad t \in \mathbb{R},
$$

and

$$
\rho_{2}(t)= \begin{cases}e^{t} & \text { if } t \leq 0 \\ 1 & \text { if } t>0\end{cases}
$$

Then we have

$$
\frac{2 r}{e} \leq \mu([-r, r])=\int_{-r}^{r} e^{\sin (t)} d t \leq 2 e r .
$$

We now prove that $\mu \in \mathcal{M}$ satisfies condition (M.1). Indeed,

$$
\sin (\tau+a) \leq 2+\sin (a) \text { for all } \tau \in \mathbb{R}, a \in A,
$$

which implies that

$$
\mu(\tau+A) \leq e^{2} \mu(A) \quad \text { for all } \tau \in \mathbb{R}
$$

so by [5], $v \in \mathcal{M}$ satisfies condition (M.1). Since

$$
\limsup _{r \rightarrow+\infty} \frac{\mu([-r, r])}{v([-r, r])}=\limsup _{r \rightarrow+\infty} \frac{\int_{-r}^{r} \rho_{1}(t) d t}{\int_{-r}^{r} \rho_{2}(t) d t}<\infty,
$$

it follows that condition (M.2) is also satisfied. We set

$$
\begin{aligned}
& \left(d_{i j}(t)\right)_{1 \leq i, j \leq 2}=\left(\begin{array}{cc}
\frac{2 \sin t+e^{-t}}{10} & \frac{\cos t}{10} \\
\frac{\sin \sqrt{2} t+e^{-t}}{10} & \frac{2 \cos \sqrt{2} t+e^{-t}}{10}
\end{array}\right), \\
& \left(a_{i j}(t)\right)_{1 \leq i, j \leq 2}=\left(\begin{array}{cc}
\frac{\cos t+e^{-t}}{10} & \frac{\sin t}{10} \\
\frac{4 \cos t+e^{-t}}{10} & \frac{\sin t+e^{-t}}{10}
\end{array}\right), \\
& \left(I_{i}(t)\right)_{1 \leq i, j \leq 2}=\left(\begin{array}{c}
\frac{8 \cos \sqrt{5} t}{10} \\
\frac{5 \sin t+e^{-t}}{10}
\end{array}\right),
\end{aligned}
$$




$$
\begin{aligned}
& \left(b_{1 j l} l(t)\right)_{1 \leq j, l \leq 2}=\left(\begin{array}{lc}
0 & \frac{3 \sin \sqrt{3} t+e^{-t}}{10} \\
0 & 0
\end{array}\right), \\
& \left(b_{2 j l}(t)\right)_{1 \leq j, l \leq 2}=\left(\begin{array}{cc}
0 & \frac{2 \cos \sqrt{5} t+e^{-t}}{10} \\
0 & 0
\end{array}\right) .
\end{aligned}
$$

Therefore

$$
L=\frac{4}{10}, \quad p_{1}=\frac{75}{100}<1, \quad \text { and } \quad q_{1}=\frac{9}{10}<1 .
$$

Using Theorems 3.8 and 3.10, we can now see that model (4.2) has a unique $(\mu, v)$-pap solution which is globally exponentially stable on

$$
\mathbb{G}=\left\{\varphi \in \mathcal{P} \mathcal{A P}\left(\mathbb{R}, \mathbb{R}^{n}, \mu, \nu\right):\left\|\varphi-\varphi_{0}\right\|_{\infty} \leq \frac{12}{10}\right\}
$$

\section{Funding}

Supported by the Slovenian Research Agency grants P1-0292, N1-0114, N1-0083, N1-0064, and J1-8131.

\section{List of abbreviations}

ap, almost periodic; apu, almost periodic uniformly; pap, pseudo almost periodic; papu, pseudo almost periodic uniformly; HOHNN, high-field Hopfield neural network.

\section{Availability of data and materials}

Not applicable.

\section{Ethics approval and consent to participate}

Not applicable.

\section{Competing interests}

Both authors declare that they have no competing interests.

\section{Consent for publication}

Not applicable.

Authors' contributions

Both authors contributed equally to the writing of this paper. Both authors have read and approved the submitted manuscript.

\section{Author details}

'IPEIK, University of Kairouan, Kairouan, Tunisia. ${ }^{2}$ LR11ES53, FSS, University of Sfax, Sfax, Tunisia. ${ }^{3}$ Faculty of Education, University of Ljubljana, Ljubljana, Slovenia. ${ }^{4}$ Faculty of Mathematics and Physics, University of Ljubljana, Ljubljana, Slovenia. ${ }^{5}$ Institute of Mathematics, Physics and Mechanics, Ljubljana, Slovenia.

\section{Publisher's Note}

Springer Nature remains neutral with regard to jurisdictional claims in published maps and institutional affiliations.

Received: 17 December 2019 Accepted: 26 February 2020 Published online: 06 March 2020

\section{References}

1. Ait Dads, E.H., Ezzinbi, K., Miraoui, M.: $(\mu, v)$-Pseudo almost automorphic solutions for some nonautonomous differential equations. Int. J. Math. 26(11), Article ID 1550090 (2015)

2. Alimi, A.M., Aouiti, C., Chérif, F., M'hamdi, M.S.: Dynamics and oscillations of generalized high-order Hopfield neural networks with mixed delays. Neurocomputing 321, 274-295 (2018)

3. Aouiti, C., M'hamdi, M.S., Chérif, F.: The existence and the stability of weighted pseudo almost periodic solution of high-order Hopfield neural network. In: International Conference on Artificial Neural Networks, pp. 478-485 (2016)

4. Arbi, A., Chérif, F., Aouiti, C., Touati, A.: Dynamics of new class of Hopfield neural networks with time-varying and distributed delays. Acta Math. Sci. 36(3), 891-912 (2016)

5. Blot, J., Cieutat, P., Ezzinbi, K.: New approach for weighted pseudo almost periodic functions under the light of measure theory, basic result and applications. Appl. Anal. 92(3), 493-526 (2013)

6. Bochner, S.: Continuous mappings of almost automorphic and almost periodic functions. Proc. Natl. Acad. Sci. USA 52, 907-910 (1964) 
7. Brahmi, H., Ammar, B., Chérif, F., Alimi, A.M.: Stability and exponential synchronization of high-order Hopfield neural networks with mixed delays. Cybern. Syst. 48(1), 49-69 (2016)

8. Chérif, F., Miraoui, M.: New results for a Lasota-Wazewska model. Int. J. Biomath. 12(2), Article ID 1950019 (2019)

9. Cieutat, P., Fatajou, S., N'Guérékata, G.M.: Composition of pseudo almost periodic and pseudo almost automorphic functions and applications to evolution equations. Appl. Anal. 89(1), 11-27 (2010)

10. Diagana, T: Weighted pseudo almost periodic solution to some differentiable equations. Nonlinear Anal. 68 , 2250-2260 (2008)

11. Diagana, T., Ezzinbi, K., Miraoui, M.: Pseudo almost periodic and pseudo-almost automorphic solutions to some evolution equations involving theoretical measure theory. CUBO 16(2), 1-31 (2014)

12. Ezzinbi, K., Miraoui, M.: $\mu$-Pseudo almost periodic and automorphic solutions in the $\alpha$-norm for some partial functional differential equations. Numer. Funct. Anal. Optim. 36(8), 991-1012 (2015)

13. Ezzinbi, K., Miraoui, M., Rebey, A.: Measure pseudo-almost periodic solutions in the $\alpha$-norm to some neutral partial differential equations with delay. Mediterr. J. Math. 13(5), 3417-3431 (2016)

14. M'hamdi, M.S., Aouiti, C., Touati, A., Alimi, A.M., Snasel, V.: Weighted pseudo almost-periodic solutions of shunting inhibitory cellular neural networks with mixed delays. Acta Math. Sci. 36(6), 1662-1682 (2016)

15. Miraoui, M.: Existence of $\mu$-pseudo almost periodic solutions to some evolution equations. Math. Methods Appl. Sci. 40(13), 4716-4726 (2017)

16. Miraoui, M.: Pseudo almost automorphic solutions for some differential equations with reflection of the argument. Numer. Funct. Anal. Optim. 38(3), 376-394 (2017)

17. Miraoui, M., Yaakoubi, N.: Measure pseudo almost periodic solutions of shunting inhibitory cellular neural networks with mixed delays. Numer. Funct. Anal. Optim. 40(5), 571-585 (2019)

18. N'Guérékata, G.M.: Almost Automorphic and Almost Periodic Functions in Abstract Spaces. Kluwer Academic, New York (2001)

19. Papageorgiou, N.S., Radulescu, V.D., Repovš, D.D.: Periodic solutions for a class of evolution inclusions. Comput. Math. Appl. 75, 3047-3065 (2018)

20. Papageorgiou, N.S., Radulescu, V.D., Repovš, D.D.: Periodic solutions for implicit evolution inclusions. Evol. Equ. Control Theory 8(3), 621-631 (2019)

21. Papageorgiou, N.S., Radulescu, V.D., Repovš, D.D.: Nonlinear Analysis-Theory and Methods. Springer Monographs in Mathematics. Springer, Cham (2019)

22. Qiu, F., Cui, B., Wu, W.: Global exponential stability of high order recurrent neural network with time-varying delays. Appl. Math. Model. 33, 198-210 (2009)

23. Radulescu, V.D., Repovš, D.D.: Partial Differential Equations with Variable Exponents. Variational Methods and Qualitative Analysis. Chapman \& Hall/CRC, Boca Raton (2015)

24. Xiao, B., Meng, H.: Existence and exponential stability of positive almost-periodic solutions for high-order Hopfield neural networks. Appl. Math. Model. 33, 532-542 (2009)

25. Yu, Y., Cai, M.: Existence and exponential stability of almost periodic solutions for high-order Hopfield neural networks. Math. Comput. Model. 47, 943-951 (2008)

26. Zhang, C.Y.: Pseudo almost periodic solutions of some differential equations. J. Math. Anal. Appl. 151, $62-76$ (1994)

27. Zhao, H.Y.: Pseudo almost periodic solutions for a class of differential equation with delays depending on state. Adv Nonlinear Anal. 9, 1251-1258 (2020)

\section{Submit your manuscript to a SpringerOpen ${ }^{\circ}$ journal and benefit from:}

- Convenient online submission

- Rigorous peer review

- Open access: articles freely available online

- High visibility within the field

- Retaining the copyright to your article

Submit your next manuscript at $\gg$ springeropen.com 